\title{
Una Aduana Digital y Sin Papeles
}

\author{
A Digital and Paper-free Customs
}

\section{RESUMEN}

La Era de la Aduana Digital se basa en una Aduana sin papeles debido al aprovechamiento de las tecnologías en sustitución del papel como requisito fundamental para ejercer su potestad aduanera y ofrecer un servicio con estándares internacionales. En otras palabras, es el cambio de mentalidad, en forma progresiva, de todos los operadores de comercio exterior que integran la cadena logística. Dicho cambio se comenzó a implementar desde el año 2012 con el Proyecto "Sistema de Despacho Aduanero" (SDA) y que a la fecha cuenta con 3 componentes:

1. SEIDA (Sistema Electrónico de Intercambio de Documentos Aduaneros): Que permite a los diversos operadores a transmitir vía electrónica sus diferentes transacciones.

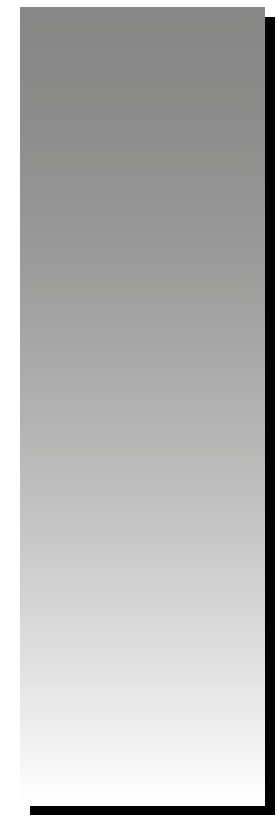

José Antonio La Noire Núñez ${ }^{1}$

Antoniolanoire@aduanua.com

Aduana

2. Portal del Operador (Internet): Es un componente de interoperabilidad entre Aduanas y los Operadores de Comercio Exterior (OCE) a través de la "Clave SUNAT Operaciones en Línea" (Clave SOL).

3. Portal del Funcionario Aduanero (Intranet): Donde los especialistas, oficiales de aduanas y jefes de unidad interactúan con flujos de trabajo y gestionan su cartera de operaciones.

Palabras claves: Aprovechamiento; tecnologías; sustitución del papel.

\section{ABSTRACT}

The era of Digital Customs is based on a paperless Customs due to the use of technologies to replace paper as a fundamental requirement to exercise its customs authority and offer a service with international standards. In other words, it is the change of mentality, progressively, of all the foreign trade operators that make up the logistics chain.

This change began to be implemented since 2012 with the "Customs Dispatch System" (CDS) Project and to date has 3 components:

1. SEIDA (Electronic System for the Exchange of Customs Documents): This allows the various operators to electronically transmit their different transactions.

2. Operator Portal (Internet): Is an interoperability component between customs and foreign trade operators through the SOL key.

3. Customs Officer Portal (Intranet): Where specialists, customs officers and unit heads interact with workflows and manage their operations portfolio.

Keywords: Exploitation; technologies; paper replacement.

\footnotetext{
${ }^{1}$ Agente de Aduana

(C) Los autores. Este artículo es publicado por Gestión en el Tercer Milenio de la Facultad de Ciencias Administrativas de la Universidad Nacional Mayor de San Marcos. Este es un artículo de acceso abierto, distribuido bajo los términos de la licencia Creative Commons Atribucion - No Comercia_Compartir Igual 4.0 Internacional. (http://creativecommons.org/licenses/by-nc-sa/4.0/) que permite el uso no comercial, distribución y reproducción en cualquier medio, siempre que la obra original sea debidamente citada.
} 


\section{INTRODUCCIÓN}

El Secretario General de la Organización Mundial de Aduanas (OMA), Kunio Mikuriya, anunció que el año 2016 estaría dedicado a la promoción de la digitalización de los procesos aduaneros, bajo el eslogan "Aduana Digital: Compromiso Progresivo" y los Miembros de la "OMA" tendrían la oportunidad de promover el uso de la información y las Tecnologías de la Información (TIC) y la Comunicación en forma progresiva.

El término de "Aduana Digital" se refiere a que el conjunto de facultades y atribuciones que tiene la Administración Aduanera para controlar el ingreso, permanencia, traslado y salida de personas, mercancías y medios de transporte, dentro del territorio aduanero será mediante actividades automatizada o electrónica que contribuyan a que la Administración Aduanera, en ejercicio de la potestad aduanera, podrá disponer la ejecución de acciones de control, antes y durante el despacho de las mercancías, con posterioridad a su levante. A esto hay que agregarle conceptos como: la Ventanilla Única de Comercio Exterior (VUCE), el uso de sistemas de intercambio de información en tiempo real, las aplicaciones de la "Nube" de acceso fácil y seguro a la información desde cualquier terminal, plataformas web, etc.

En esta nueva era de la "Aduana Digital" se ha transformado la forma en que se opera debido a la mayor capacidad de comunicación que se traduce en: simplificación de procesos, intercambio información, mejor coordinación de acciones Prevención del Contrabando y Control Fronterizo. Es decir, las tecnologías han tenido un impacto positivo en la operatividad de la Aduana debido a que han optimizado su labor mediante:

- La difusión en el portal web de la Superintendencia Nacional de Aduanas y de Administración Tributaria (SUNAT) de toda la información sobre procedimientos, informes, tratados y funciones, así como la difusión de los servicios que ofrece;

- La optimización de los tiempos de despacho, se debe en gran parte a la eliminación paulatina de la presentación física de algunos documentos aduaneros y de presentación física de ciertos expedientes a través de la implementación del Portal de Operador.

- Una mejora en la coordinación entre las intendencias de las Aduanas operativas dentro del territorio aduanero;

- La numeración de la Declaración Aduanera es a través de medios electrónicos y es la misma Administración, la que determinará cuando se presentará por escrito. Dicha Declaración puede ser rectificada y/o legajada de la misma forma, dependiendo del régimen, la intendencia y la situación del despacho;

- El uso de medición de desempeño para detectar y solucionar los cuellos de botella de procedimientos y mejorar la competitividad como país;

- Asimismo, con la remisión del informe Anual del Oficial de Cumplimiento (IAOC) que de acuerdo con la norma de la Unidad de Inteligencia Financiera (UIF), se envía desde el Portal de Prevención de Lavado de Activos y del Financiamiento del Terrorismo (plaft.sbs.gob.pe), el cual constituye el único medio para el envío del IAOC a la UIF-Perú.

\section{OBJETIVO DEL ENSAYO}

El objetivo del ensayo es invitar a todos los interesados a mirar hacia el futuro y entender que la función de la SUNAT, no es la de controlar y fiscalizar, sino la de facilitar y supervisar el tráfico de mercancías, personas y medios de transporte, con los mejores cuidados y tiempos. Dicho de otra manera, que el ciudadano de a pie reconozca los esfuerzos de mejora de la SUNAT como funciones propias de un Ente encargado de administrar Tributos Internos y Aduanas.

\section{ARGUMENTACIÓN}

La segunda década del siglo XXI nos ofrece un cambio de perspectiva en lo económico, político y social, y es que gracias la transparencia del uso de recursos tecnológicos se ha puesto en evidencia desde temas de corrupción de funcionarios hasta una serie de mejoras en los servicios, no sólo privados, sino públicos; y la SUNAT no podría quedar exenta de estos cambios. Ahora bien, si hiciéramos un recopilatorio 
de todos los cambios que ha experimentado la legislación Aduanera peruana en estas últimas dos décadas no podríamos de dejar de mencionar estas funciones $\mathrm{y} / \mathrm{u}$ objetivos publicados en la Memoria Anual 2015 de la SUNAT:

- Mejorar en el cumplimiento voluntario,

- Reducir los costos y tiempos para el usuario,

- Elaborar y fortalecer planes regionales de desarrollo sobre gestión de riesgos aduaneros,

- Colaborar con el sector privado y con otros órganos gubernamentales. De esta forma se cumple el "Marco de Estándares para Asegurar y Facilitar el Comercio" de la OMA, conocido como "Marco SAFE", por sus siglas en inglés.

- Marco de Estándares para Asegurar y Facilitar el Comercio

- Garantizar la seguridad de la cadena logística,

- Reducir el contrabando y el tráfico Ilícito de mercancías,

- Asegurar el fortalecimiento institucional,

- Desarrollar el recurso humano,

- Simplificar procedimientos,

- Celebrar acuerdos y convenios de cooperación técnica y administrativa.

Para hablar de los cambios en la Legislación Aduanera Peruana, podemos mencionar el ocurrido apenas entrando en la segunda década del siglo XXI y es la promulgación de la Nueva y actual Ley General de Aduanas, con el Decreto Legislativo № 1053, dicha Ley, que no tiene una antigüedad mayor de 10 años, debe ser entendida como consecuencia de las negociaciones del "Acuerdo de Promoción Comercial entre Perú y Estados Unidos" (APC Perú EE.UU); es decir, que Estados Unidos le exigió al Perú que para negociar un Tratado de Libre Comercio (TLC) primero debían cambiar su legislación por considerarla poco competitiva y/o inadecuada para la dinámica del Comercio Exterior. Además que, con la subsiguiente modificatoria a la Ley, el Decreto Legislativo № 1122, publicada el año 2012, se incorpora la figura del Operador Económico Autorizado (OEA), figura que toma total relevancia ya que por primera vez en su historia la SUNAT divide, valida y reconoce que en el mercado nacional de Operadores de Comercio Exterior (OCE) existen de 02 categorías, los que son OEA y los que No, al otorgar una certificación de Nivel Internacional a un OCE. Posteriormente, se han seguido publicando una serie de modificaciones, debido a que toda legislación es perfectible en el tiempo, tanto a la Ley como al Reglamento mediante la publicación de:

- La Ley № 30038, publicada el 07/06/2013,

- El Decreto Legislativo № 1235, publicado el 26/09/2015,

- La Ley № 30498, publicada el 08/08/2016,

- El Decreto Legislativo № 1263 publicado el 10/12/2016, y

- La última modificación a la Ley, el Decreto Legislativo № 1433 publicado recientemente el 16/09/2018, que contempla en sus disposiciones finales una próxima modificación al reglamento; como una manera de adecuarnos al Programa de Facilitación Aduanera, Seguridad y Transparencia (FAST) de la Organización Mundial de Comercio (OMC).

Además de la publicación de una serie de informes por la Intendencia Nacional Jurídico Aduanera de la SUNAT y de modificaciones y/o simplificaciones de procedimientos, en respuesta a una serie de interrogantes formuladas por los mismos participantes de la cadena logística, así como de gremios que, con el solo uso de medios electrónicos, pueden cumplir con sus obligaciones propias de cada operador:

- La Inspección no intrusiva, inspección física y reconocimiento físico de mercancías en el Complejo Aduanero "Sistema de Inspección No Intrusiva” (SINI) de la Intendencia de Aduana Marítima del Callao. Establecidas sus pautas a seguir mediante el Procedimiento Específico de Fiscalización y Prevención del Contrabando y Control Fronterizo (CONTROL-PE.00.09). 
- El Informe $\mathrm{N}^{\circ}$ 169-2018-SUNAT/340000 sobre la "no exigibilidad de la impresión del formato original y copia de la Declaración Aduanera de Mercancías (DAM)".

- El Informe $\mathrm{N}^{\circ} 156$-2018-SUNAT/340000 sobre la posibilidad de que "el agente de aduana puede suscribir en nombre del importador el formato B de la DAM".

- El Procedimiento Específico de "Mandato Electrónico" (DESPA-PE.00.18), que otorga mandato tanto al importador y exportador mediante la validación del dueño e interesado de la carga a través de su Clave SOL en el portal del operador (todavía en implementación para los demás regímenes aduaneros).

- El Procedimiento Específico de "Registro del Personal de los Operadores de Comercio Exterior" (DESPA-PE.24.04), que anula la presentación física en ventanilla de documentación para la acreditación de ciertos trabajadores de cada operador (se establecen los supuestos y se acreditan a través del portal del Operador).

- La Resolución № 020-2018: SUNAT, que establece la obligación de emitir de manera electrónica facturas, boletas y notas en Declaraciones Aduaneras de Exportación.

Siguiendo con el tema de Cooperación Internacional, tenemos que el año 2014 entre el 23 y 25 de abril nuestro país fue sede de la XVII Conferencia Regional de Directores Generales de Aduanas de las Américas y el Caribe (CRD$\mathrm{GA}$ ), que reunió a representantes de más de 30 países miembros de la OMA, y que la SUNAT fue la anfitriona.

El objetivo fundamental del foro fue, entre otros, avanzar en la facilitación del comercio exterior sin renunciar a un eficiente control aduanero a través de:

- La puesta en marcha de la plataforma virtual "Sistema de Despacho Aduanero" (SDA), desarrollada por la SUNAT para gestionar el intercambio de información $y$ el registro de operaciones de comercio exterior.
Con la aplicación de este sistema la SUNAT se coloca como una Administración de clase mundial y nos coloca en la ruta de las aduanas del siglo XXI. De esta forma, el Perú cumple con los retos propuestos en el Acuerdo de Facilitación de Comercio de la OMC en el Programa de Facilitación Aduanera, Seguridad y Transparencia (FAST) que actualmente viene desarrollando la SUNAT y que plantea facilitar, agilizar, automatizar y transparentar los procesos de entrada y salida de mercancías en las aduanas del país, así como en los procesos complementarios del control de operadores.

\section{CONCLUSIONES}

- Se evidencia una reducción en la generación de residuos y en el consumo de recursos naturales al eliminar procesos burocráticos redundantes al aplicar criterios eco-amigables para el requerimiento de los diferentes tipos de documentos para el cumplimiento del conjunto de formalidades aduaneras. De esta forma, se evita la falta de control por perdida de documentación.

- Con el registro en línea a través de la Clave SOL se reducen los ciclos de espera, se simplifican procesos que se ve reflejado en un aprovechamiento en el costo-oportunidad de los Operadores de Comercio Exterior. El uso medios tecnológicos en el manejo de la información, es la consecuencia de la aplicación de los principios generales del derecho aduaneros, como son la transparencia, la facilitación y la modernización.

- Que el contribuyente, usuario y/u Operador de Comercio Exterior reconozca que la SUNAT es la encargada de administrar la política fiscal y es el eje central de la Política comercial del Perú para con el resto del mundo, bajo estándares internacional de calidad, eficiencia y de colaboración con el medio ambiente.

\section{AGRADECIMIENTOS Y RECONOCIMIENTOS}

A mi madre

\section{REFERENCIAS BIBLIOGRÁFICAS}

Portal Institucional de la SUNAT. "Ley General de Aduanas, su reglamento y modificatorias". Re- 
cuperado de http://www.sunat.gob.pe/legislacion/aduanera/index.html

Portal Institucional de la SUNAT. "Programa Facilitación Aduanera, Seguridad y Transparencia" (FAST). Recuperado de http://www.sunat.gob. pe/operatividadaduanera/fast/index.html

Mikuriya, kunio (2016). “Mensaje de la Organización Mundial de Aduanas Día Internacional de la Aduana 2016". Recuperado de http://www. wcoomd.org/ /media/wco/public/global/pdf/ about-us/international-customs-day/2016/ wco-sg-message-icd-2016-es.pdf?db=web

Agencia Peruana de Noticias Andina. Publicado el 22/08/2018 en Lima, Perú. "SUNAT continúa simplificando operaciones de Comercio Exterior". Recuperado de https://andina.pe/agencia/ noticia-sunat-continua-simplificando-operaciones-comercio-exterior-722840.aspx

Redacción Gestión. Publicado el 22/04/2014 en Lima, Perú. "SUNAT: en la ruta de las Aduanas del Siglo XXI". Recuperado de https:// gestion.pe/impresa/sunat-ruta-aduanas-siglo-xxi-58010

Portal Institucional de la SUNAT. "Proyecto Sistema de Despacho Aduanero" (SDA). Recuperado de http://www.sunat.gob.pe/operatividadaduanera/ sda/index.htmlVélez, G.; Correa, L.; Duarte, J.; Stuarte, D. (2005). Conocimiento gerencial requerido del enfermero en el programa salud de la familia, Brasil.

Yáñez, M.; Ávila, J.; Bermúdez, M.; Bellver, V.; Guilabert, M.; Mira, J. (2016). Capacidad de gestión de los ejecutivos de enfermería, España. 
